\title{
A comparison between plaque-based and vessel-based measurement for plaque component using volumetric intravascular ultrasound radiofrequency data analysis
}

\author{
Eun-Seok Shin • Hector M. Garcia-Garcia • \\ Scot Garg • Patrick W. Serruys
}

Received: 28 December 2009/Accepted: 28 August 2010/Published online: 11 September 2010

(C) Springer Science+Business Media, B.V. 2010

\begin{abstract}
Although percent plaque components on plaque-based measurement have been used traditionally in previous studies, the impact of vessel-based measurement for percent plaque components have yet to be studied. The purpose of this study was therefore to correlate percent plaque components derived by plaque- and vessel-based measurement using intravascular ultrasound Virtual Histology (IVUS-VH). The patient cohort comprised of 206 patients with de novo coronary artery lesions who were imaged with IVUSVH. Age ranged from 35 to 88 years old, and 124 patients were male. Whole pullback analysis was used to calculate plaque volume, vessel volume, and absolute and percent volumes of fibrous, fibrofatty, necrotic core, and dense calcium. The plaque and vessel volumes were well correlated $(r=0.893$, $P<0.001)$. There was a strong correlation between percent plaque components volumes calculated by plaque and those calculated by vessel volumes (fibrous; $r=0.927, P<0.001$, fibrofatty; $r=0.972$, $P<0.001$, necrotic core; $r=0.964, P<0.001$, dense calcium; $r=0.980, P<0.001$,). Plaque and vessel
\end{abstract}

\author{
E.-S. Shin $(\bowtie)$ \\ Ulsan University Hospital, \\ University of Ulsan College of Medicine, \\ 290-3 Jeonha-dong, Dong-gu, \\ Ulsan 682-714, South Korea \\ e-mail: ses@uuh.ulsan.kr
}

H. M. Garcia-Garcia · S. Garg · P. W. Serruys

Thoraxcenter, Erasmus MC, Rotterdam, The Netherlands volumes correlated well to the overall plaque burden. For percent plaque component volume, plaque-based measurement was also highly correlated with vesselbased measurement. Therefore, the percent plaque component volume calculated by vessel volume could be used instead of the conventional percent plaque component volume calculated by plaque volume.

Keywords Atherosclerosis - Intravascular ultrasound · Virtual histology

\section{Introduction}

The absolute and percent plaque component volumes derived by plaque-based measurement have been studied traditionally in vivo coronary artery disease using intravascular ultrasound Virtual Histology (IVUS-VH) [1-6]. Although a recent paper showed a moderate relationship between the area of each plaque component and overall plaque area [7], the impact of vessel size on the absolute and percent plaque component content has yet to be studied.

During the early stages of coronary atherosclerosis it is well known that human coronary arteries enlarge in relation to plaque area, and thus a functionally important luminal stenosis may be delayed [8]. We hypothesize that plaque size is closely related to vessel size, and thus the percent plaque component derived by plaque-based measurement will be related to those derived by vessel-based measurement. The purpose of this study was to correlate percent plaque 
components volumes calculated by plaque and those calculated by vessel volumes as assessed by IVUS$\mathrm{VH}$.

\section{Methods}

Study patients

We have selected the patients retrospectively. All patients included that were imaged with IVUS-VH were considered if they had sufficient quality to be analyzed between August 2005 and September 2007. This study enrolled 206 consecutive patients with de novo coronary artery lesion, who presented with stable angina or acute coronary syndrome, defined as unstable angina or non ST-segment elevation myocardial infarction. Patients with left main disease, chronic total occlusions or intracoronary thrombus in the target vessel on angiography were excluded. No complications occurred related to the IVUS imaging. The culprit lesion was defined as the coronary lesion thought responsible for the patient's symptoms that was treated with percutaneous coronary intervention. All patients provided written informed consent and the study was approved by the hospital's institutional review board.

IVUS-VH examination and analysis

IVUS-VH creates color coded tissue maps to classify atherosclerotic plaques into 4 major components (fibrous: green; fibrofatty: light-green; necrotic core: red; and dense calcium: white) using commercially available phased-array IVUS catheters (Eagle Eye Gold 2.9F $20 \mathrm{MHz}$ ) connected to a dedicated IVUSVH console (Volcano Corporation, Rancho Cordova, USA). Image acquisition is ECG-gated. After intracoronary injection of $200 \mu \mathrm{g}$ nitroglycerine, continuous pullback of the IVUS catheter was performed using a motorized pullback device at $0.5 \mathrm{~mm} / \mathrm{s}$. IVUS-VH data from the pullback was stored for off-line analysis by an independent core laboratory (Cardialysis BV, Rotterdam, The Netherlands). The lesion comprised of the most diseased $10 \mathrm{~mm}$ segment that contained the largest plaque burden [9]. Off-line volumetric reconstruction of this segment was performed using pcVH 2.2 (Volcano Corporation). The contours of each cross sectional image per frame were manually corrected. The lumen contour was detected by tracing the leading edge of the intima, and the vessel contour by tracing the leading edge of the adventitia. For each segment, geometrical and compositional analysis was performed. In addition, percent plaque components volumes to vessel volume were calculated as plaque components volumes divided by vessel volume. Plaque burden defined as plaque plus media volume divided by vessel volume $\times 100$. Mean lumen area calculated by dividing lumen volume by lesion length.

Data analysis

The following correlations were performed in the overall population:

(1) Plaque volume to vessel volume

(2) Absolute plaque component volume to plaque volume

(3) Absolute plaque component volume to vessel volume

(4) Percent plaque component volume calculated by plaque volume to percent plaque component volume calculated by vessel volume

Statistical analysis

All analyses were done using SPSS (version 17.0; SPSS Inc, Chicago, Illinois). Data are presented as frequencies or mean \pm SD. The Pearson's correlation coefficient was used to assess associations between measured parameters. A two-sided $P$-value $<0.05$ was considered significant.

\section{Results}

Table 1 shows patients' characteristics. The mean age was 61 years (range 35-88), and 124 (61.1\%) patients were male. Clinical presentation was the stable angina in 88 patients $(42.7 \%)$ and acute coronary syndrome in $118(57.3 \%)$. Table 2 presents the geometrical and compositional analyses using IVUS-VH. The plaque burden was $52.8 \%$ and lesion length was $10.8 \mathrm{~mm}$. Overall, the predominant plaque composition was fibrous tissue $\left(31.9 \pm 19.3 \mathrm{~mm}^{3}\right)$ followed by necrotic core $\left(12.1 \pm 9.0 \mathrm{~mm}^{3}\right)$, fibrofatty $(5.9 \pm 5.7$ $\left.\mathrm{mm}^{3}\right)$, and dense calcium $\left(5.7 \pm 5.7 \mathrm{~mm}^{3}\right)$. 
Table 1 Baseline characteristics

\begin{tabular}{ll}
\hline & $N=206$ (Lesions = 246) \\
\hline Age (year) & $61.0 \pm 9.5$ \\
Male & $124(61.1)$ \\
Diabetes & $56(27.2)$ \\
Hypertension & $114(55.3)$ \\
Current smoker & $53(25.7)$ \\
Hypercholesterolemia & $112(55.2)$ \\
Clinical state & \\
Stable angina & $88(42.7)$ \\
Acute coronary syndrome & $118(57.3)$ \\
Total cholesterol (mg/dl) & $185.1 \pm 37.1$ \\
LDL cholesterol (mg/dl) & $106.2 \pm 34.5$ \\
hiCRP (mg/L) & $2.7 \pm 4.2$ \\
Study vessel & $135(54.9)$ \\
LAD & $39(15.9)$ \\
LCX & $72(29.3)$ \\
RCA & $159(64.6)$ \\
Lesion & $87(35.4)$ \\
Culprit & \\
Non-culprit & \\
\hline Values aresented &
\end{tabular}

Values are presented as number (\%) or mean \pm SD. $L D L$ lowdensity lipoprotein, $h s C R P$ high-sensitivity C-reactive protein, $L A D$ left anterior descending artery, $L C X$ left circumflex artery, $R C A$ right coronary artery

Overall, the plaque volume was well correlated with vessel volume (correlation coefficient $r=0.893$, $P<0.001$ ), which was consistent irrespective of the plaque burden (PB): $\mathrm{PB}<40 \%(r=0.894, P<$ $0.001), 40 \leq \mathrm{PB}<50 \%$ ( $r=0.984, P<0.001), 50 \leq$ $\mathrm{PB}<60 \%(r=0.991, P<0.001), 60 \leq \mathrm{PB}<70(r=$ $0.987, P<0.001)$ and $\mathrm{PB}>70 \% \quad(r=0.990$, $P<0.001$ ) (Fig. 1). The correlation between fibrous volume and plaque volume was stronger than the correlation between fibrous volume and vessel volume. In addition, these two correlation coefficients were stronger when compared to those from the other tissue types (Fig. 2). Interestingly, in both groups there were significant correlations when comparing percent plaque components volumes calculated by plaque volume, and those calculated by vessel volume (Fig. 3).

When mean lumen area was plotted against the plaque burden, lumen was encroached on by plaque, and mean lumen area decreased in direct relation to the plaque burden (Fig. 4).
Table 2 Geometrical and compositional analysis using IVUS$\mathrm{VH}$

\begin{tabular}{lc}
\hline & Lesions $=246$ \\
\hline Geometrical measurements & \\
Vessel volume, $\mathrm{mm}^{3}$ & $176.9 \pm 62.4$ \\
Lumen volume, $\mathrm{mm}^{3}$ & $83.7 \pm 34.0$ \\
Plaque volume, $\mathrm{mm}^{3}$ & $93.1 \pm 36.5$ \\
Plaque burden, \% & $52.8 \pm 9.0$ \\
Lesion length, mm & $10.8 \pm 2.3$ \\
Compositional measurements & \\
FI volume, mm & \\
FF volume, $\mathrm{mm}^{3}$ & $31.9 \pm 19.3$ \\
$\mathrm{NC}$ volume, $\mathrm{mm}^{3}$ & $5.9 \pm 5.7$ \\
DC volume, $\mathrm{mm}^{3}$ & $12.1 \pm 9.0$ \\
Percent volume to plaque & $5.7 \pm 5.7$ \\
FI & \\
FF & $32.3 \pm 9.4$ \\
NC & $5.9 \pm 4.6$ \\
DC & $12.5 \pm 6.8$ \\
Percent volume to vessel & $6.0 \pm 5.4$ \\
FI & \\
FF & $17.5 \pm 7.2$ \\
NC & $3.3 \pm 2.9$ \\
DC & $6.7 \pm 4.1$ \\
\hline Val & $3.2 \pm 3.0$ \\
\hline
\end{tabular}

Values are presented mean \pm SD. Plaque volume included media volume. $P B$ plaque burden, $F I$ fibrous, $F F$ fibrofatty, $D C$ dense calcium, $N C$ necrotic core

In Fig. 5, there was a good correlation between plaque volume and vessel volume in patients with stable angina $(r=0.880, P<0.001)$ and acute coronary syndrome $(r=0.908, P<0.001)$.

\section{Discussion}

Plaque progression is influenced by many risk factors and its enlargement is generally the consequence of long term exposure to them. This plaque progression is followed by concomitant enlargement of the vessel, a process known as remodelling. Although expansive remodelling and plaque composition are associated with plaque vulnerability, the relationship between vessel size and plaque composition has not been studied. The major findings of this study were: (1) Plaque volume and vessel volume were well correlated irrespective of plaque burden and clinical states; (2) The percent plaque 

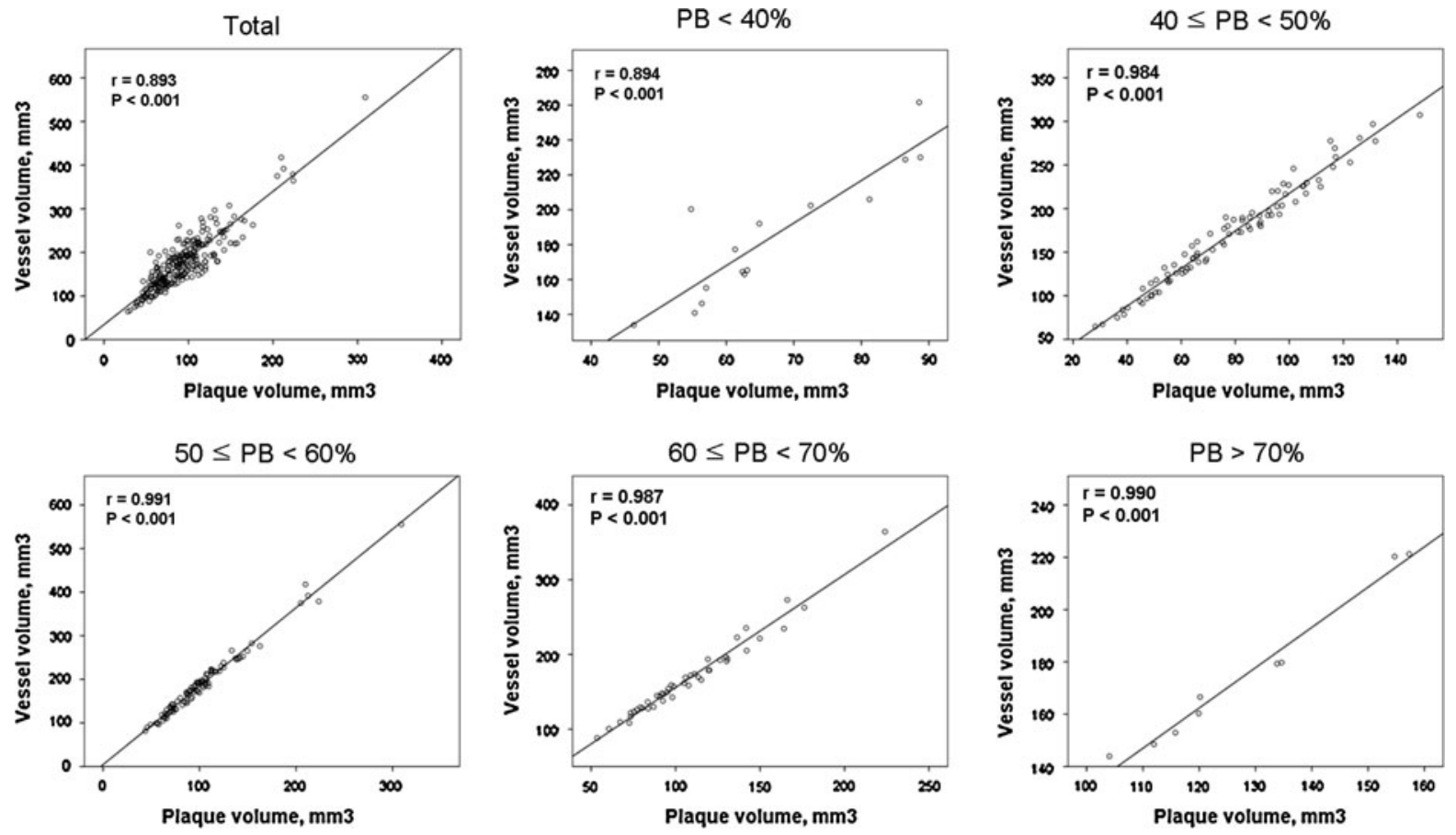

Fig. 1 Graphs show significant correlation between plaque volume and vessel volume. It is consistent with different plaque burdens $(\mathrm{PB}=$ plaque burden)

components volumes calculated by plaque volume correlated well to those calculated by vessel volume.

Qian et al. [7] recently demonstrated that the mean area of each plaque component correlated to overall plaque area, however, they did not show any relationship between the percent tissue type areas calculated by vessel areas. In the present study, using volumetric analysis, a similar correlation has been found between the mean area of each plaque component and the overall plaque area, and it is noteworthy that the percent plaque component volume calculated by plaque volume, correlated well to those calculated by vessel volume. As a matter of course the plaque volume and vessel volume were well correlated; the results being consistent irrespective of plaque burden. All four plaque components volumes to plaque volume showed a similar relation with each plaque component volume to vessel volume. These results suggest that the percent plaque component volume calculated by vessel volume can be used together with the classical parameter of the percent plaque component volume calculated by plaque volume. Of note, the use of both parameters may enable longitudinal changes to be more completely characterized.
Our findings suggest that the rate of plaque deposition exceeds the rate of vessel enlargement. Previous data demonstrate that at a greater than $40 \%$ diameter stenosis, the plaque area continues to increase to finally involve the entire circumference of the vessel, whilst the artery itself no longer enlarges at a rate sufficient to prevent narrowing of the lumen [9]. In contrast, our findings suggest that the artery and plaque size can both increase simultaneously, however the rate of plaque deposition appears to be more rapid than the rate of artery enlargement.

It has been reported that the inter-observer variability is higher for lumen contouring than for vessel contouring [10], moreover, lumen contouring is also more time consuming and challenging due to the presence of artifacts. These factors hamper the wide use of this imaging technique in the catheterization laboratory. It follows that a technique which can help overcome these problems would strengthen the position of this imaging technique in the decision making process. Recently, the Shin's method has been introduced; the method entails drawing the contour of the IVUS catheter instead of the lumen contour [11]. This method allows rapid quantification 
Fig. 2 Graphs show correlation between the absolute volume of each plaque component to plaque volume and those to vessel volume
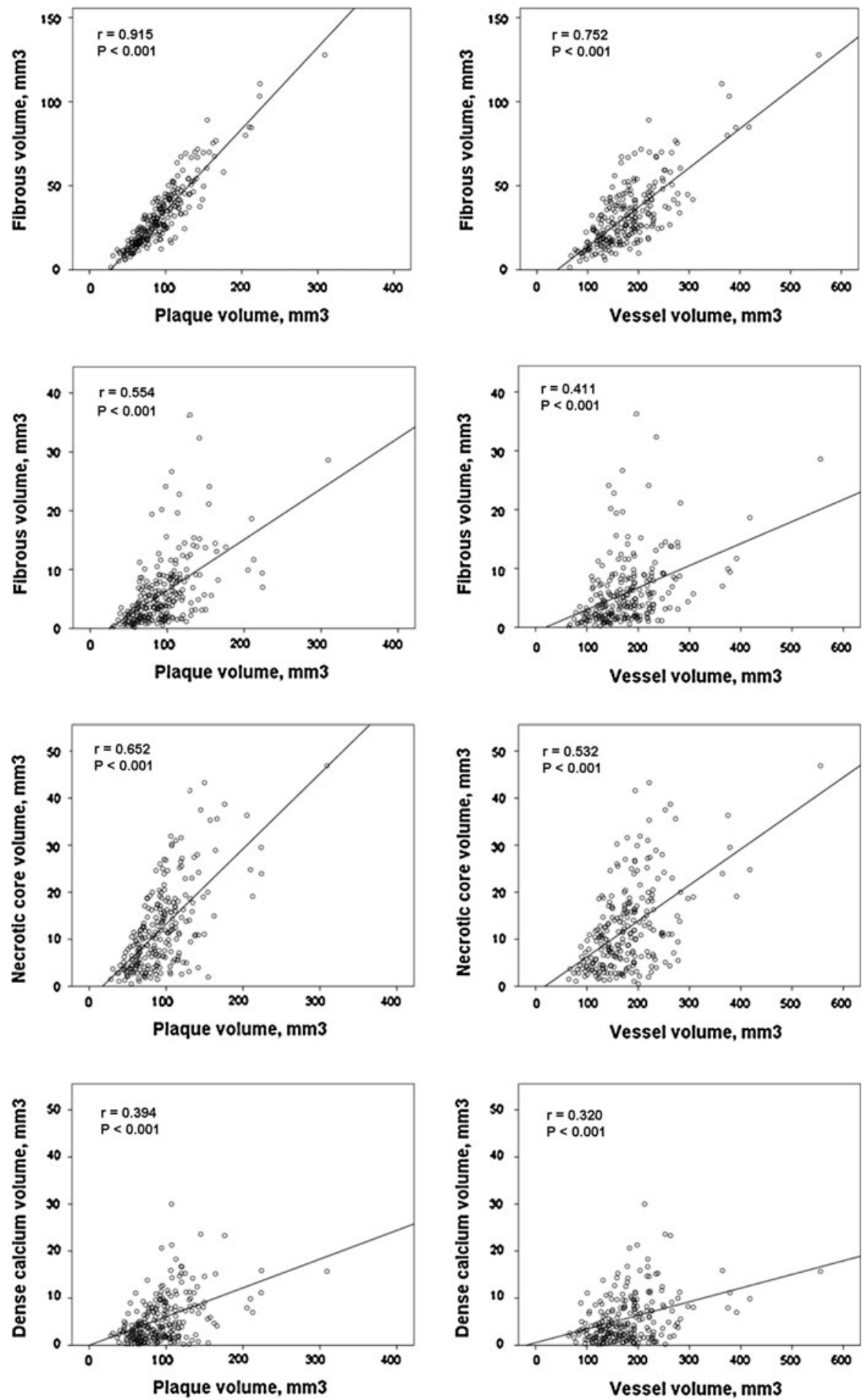

of dense calcium and necrotic core. Also it showed a good correlation with the conventional method and excellent reproducibility for measurement of necrotic core and dense calcium using IVUS-VH. The authors, therefore, said Shin's method could replace the conventional method for necrotic core and calcium 
Fig. 3 Graphs show significant correlations between the percent plaque components volumes calculated by plaque volume and those calculated by vessel volume
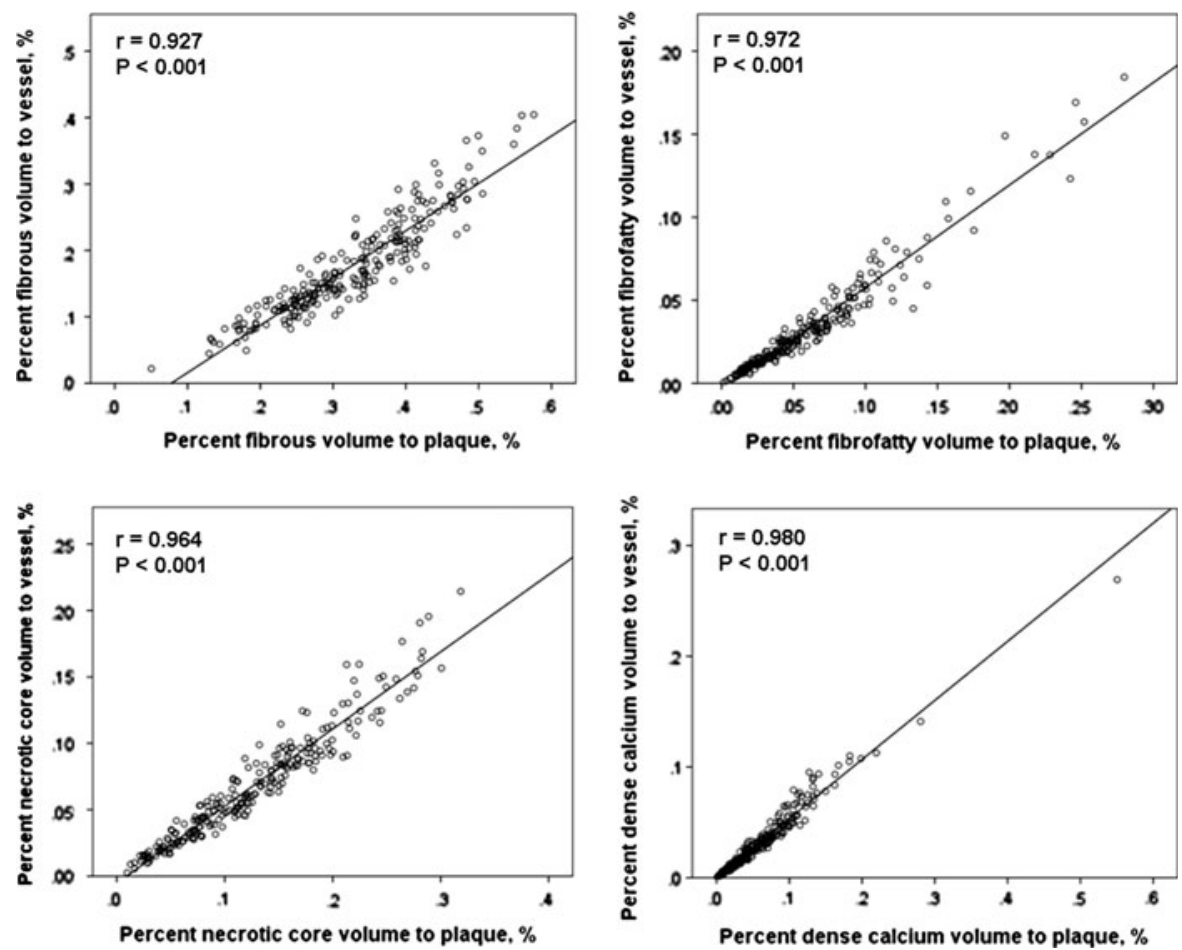

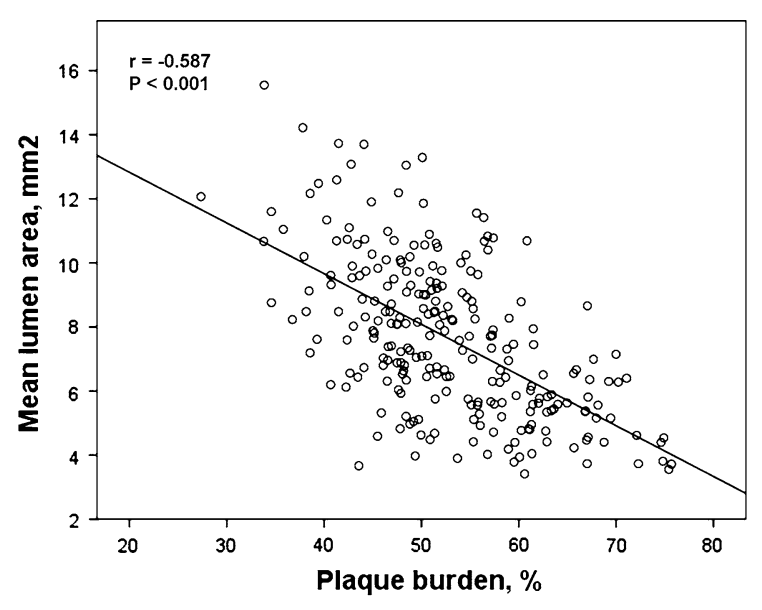

Fig. 4 Graph shows negative correlation between plaque burden, a measure of volumetric analysis and mean lumen area. Mean lumen area decreases in direct relation to the plaque burden

measurement in atherosclerotic lesions, and it might be useful in the catheterization laboratory for online clinical decision. However, due to its nature (It is not plaque-based measurement), it does not provide plaque size and percent plaque component derived by plaque-based measurement. Thus the only percentages that can be derived are those taking into account vessel size. Therefore, in this analysis we have explored the relationship between plaque-and vessel-based measurements. Importantly in the calculation of percent necrotic core, which is of crucial relevance due to its influence on plaque vulnerability, the new parameters of percent necrotic core and dense calcium volumes derived by vessel-based measurement are not affected by the under-estimation of lumen contour detection in Shin's method. In present study, it follows that the percent plaque component volume calculated by vessel volume could be a useful parameter for Shin's method to evaluate the content of necrotic core and calcium. In addition, with a shorter analysis time, Shin's method may have an important role to play in determining absolute and percent necrotic core, and dense calcium 'real-time' in the catheterization laboratory.

\section{Limitations}

We evaluated those lesions in the most diseased $10 \mathrm{~mm}$ segment of the vessel that contained the largest plaque burden. Therefore we do not know the relationship between plaque and vessel sizes in other 
Fig. 5 Graphs show significant correlation between plaque volume and vessel volume. It is consistent in stable angina and acute coronary syndrome

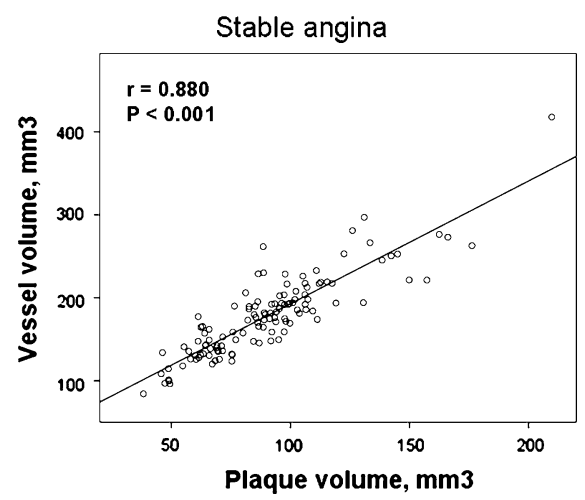

less serve atherosclerotic lesions. Of note, we did include lesions with various amount of plaque burden.

\section{Conclusions}

Plaque and vessel volumes correlated well to overall plaque burden. For percent plaque component volume, plaque-based measurement was also highly correlated with vessel-based measurement. Therefore, vesselbased measurement could replace conventional plaque-based measurement for determining the percent plaque component in atherosclerotic lesions.

Conflict of interest All authors have approved the final manuscript, which has not been published and is not under consideration elsewhere. We declare that there is no conflict of interest for any author.

\section{References}

1. Valgimigli M, Rodriguez-Granillo GA, Garcia-Garcia HM et al (2006) Distance from the ostium as an independent determinant of coronary plaque composition in vivo: an intravascular ultrasound study based radiofrequency data analysis in humans. Eur Heart J 27:655-663

2. Rodriguez-Granillo GA, Garcia-Garcia HM, Valgimigli M et al (2006) Global characterization of coronary plaque rupture phenotype using three-vessel intravascular ultrasound radiofrequency data analysis. Eur Heart J 27:19211927
3. Mehta SK, McCrary JR, Frutkin AD et al (2007) Intravascular ultrasound radiofrequency analysis of coronary atherosclerosis: an emerging technology for the assessment of vulnerable plaque. Eur Heart J 28:1283-1288

4. Hong M-K, Mintz GS, Lee CW et al (2007) Comparison of virtual histology to intravascular ultrasound of culprit coronary lesions in acute coronary syndrome and target coronary lesions in stable angina pectoris. Am J Cardiol 100:953-959

5. Garcia-Garcia HM, Gonzalo N, Regar E et al (2009) Virtual histology and optical coherence tomography: from research to a broad clinical application. Heart 95:1362-1374

6. Fujii K, Carlier ShG, Mintz GS et al (2005) Association of plaque characterization by intravascular ultrasound virtual histology and arterial remodelling. Am J Cardiol 96:14761483

7. Qian J, Maehara A, Mintz GS et al (2009) Relation between individual plaque components and overall plaque burden in the prospective, multicenter virtual histology intravascular ultrasound registry. Am J Cardiol 104:501-506

8. Glagov S, Weisenberg E, Zarins C et al (1987) Compensatory enlargement of human atherosclerotic coronary arteries. N Engl J Med 316:1371-1375

9. Serruys PW, Garcia-Garcia HM, Buszman P et al (2008) Effects of the direct lipoprotein-associated phospholipase $\mathrm{A}(2)$ inhibitor darapladib on human coronary atherosclerotic plaque. Circulation 118:1172-1182

10. Rodriguez-Granillo GA, Vaina S, Garcia-Garcia HM et al (2006) Reproducibility of intravascular ultrasound radiofrequency data analysis: implications for the design of longitudinal studies. Int J Cardiovasc Imaging 22:621-631

11. Shin ES, Garcia-Garcia HM, Serruys PW (2010) A new method to measure necrotic core and calcium content in coronary plaques using intravascular ultrasound radiofrequency-based analysis. Int $\mathrm{J}$ Cardiovasc Imaging 26: 387-396 